\title{
CONTRIBUTION A L’ÉTUDE ANATOMO-PATHOLOGIQUE
}

\section{DE L'INFECTION à PLASMODIUM BERGHEI,}

\section{CHEZ LES SOURIS BLANCHES SOUMISES À DIVERS RÉGIMES}

\author{
Par H. PAYAN, J. SAUTET et J. ALDIGHIERI
}

Dans une série d'expériences portant sur 30 lots, de chacun 15 souris blanches de 20 g., provenant de l'élevage H (Allauch), nous avons constaté que l'enrichissement du régime des souris par un apport de vitamines A et $\mathrm{D}$, seules ou par l'apport des mêmes éléments contenus dans l'huile de foie de morue, avait un effet bénéfique sur le traitement du paludisme expérimental par la chloroquine (nivaquine). Cependant, nous avions cru constater que, si cet enrichissement du régime était obtenu par une prise quotidienne d'huile de foie de morue, l'action était moins bonne que celle obtenue avec l'apport simple de quantité équivalente de vitamines A et D seules, en même temps que nous observions une chute de poids, tant que durait le régime à l'huile de foie de morue.

Nous nous sommes alors demandé si l'apport important de lipide de l'huile de foie de morue ne contribuait pas à accentuer la dégénérescence graisseuse, déjà signalée dans les affections à Plasmodium berghei, rendant ainsi son action moins favorable que ses composants vitaminiques seuls.

Nous avons alors fait des prélèvements de foie chez nos souris, ainsi que de rate, afin de suivre parallèlement les lésions classiques de cet organe.

Les résultats des observations sont les suivants, le traitement par la chloroquine étant toujours le même $(9,2 \mathrm{mg}$. par kilo, voie orale, pendant cinq jours - deux jours après l'inoculation d'une dose connue d'hématies parasitées).

Quant au régime enrichi, il débutait sept jours avant l'inoculation du Plasmodium berghei.

\section{SOURIS AVEC RÉGIME, IMPALUDÉES ET TRAITÉES}

Série 10I: Huile de foie de morue (vitamine A, 2.000 U.I. ; vitamine D, 500 U.I., $0,3 \mathrm{~cm}^{3}$ tous les 2 jours). 
- Souris morte le 6 novembre $(15$ jours $)=$ Le foie comporte une réaction küppférienne diffuse au contact de pigment, réaction assez discrète. Réaction macrophagique importante au sein du parenchyme splénique; la pulpe blanche est peu visible, les éléments de type mégacaryocytaire sont peu nombreux.

- Souris morte le 24 novembre (33 jours) = Surcharge pigmentaire importante des éléments küppfériens au sein du parenchyme hépatique ; cette réaction est diffuse, mais les dépôts pigmentaires, les plus massifs, prédominent nettement à la partie moyenne et à

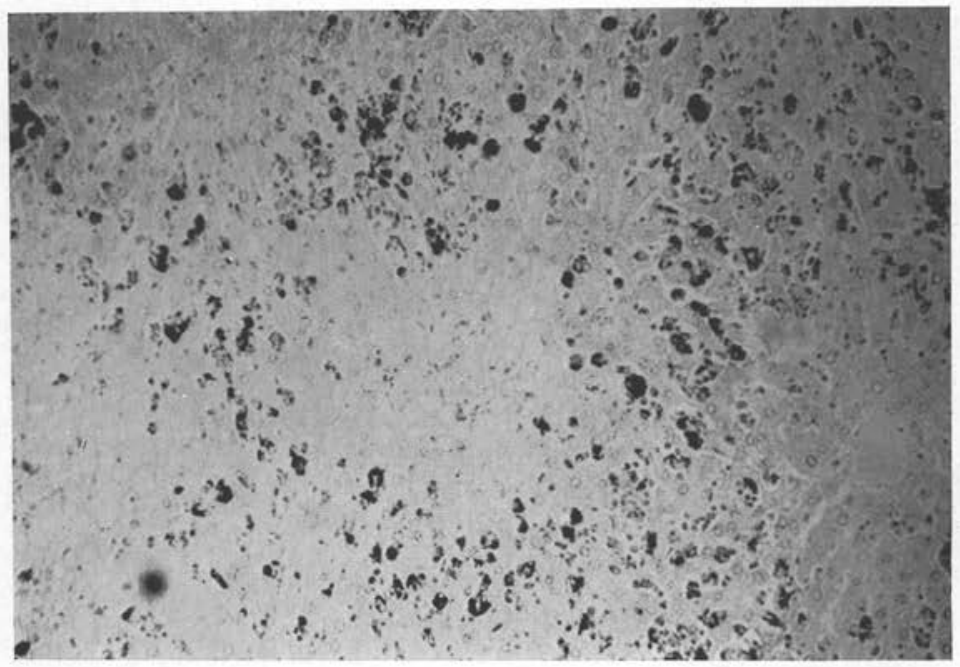

FIt. 1. - 101-24.11 (33 jours). Foie. Surcharge pigmentaire importante prédominant à la partie moyenne et périphérique des lobules, la systématisation de cette surcharge étant très nette.

la périphérie des lobules. On note par ailleurs quelques petits foyers de stéatose au sein des cellules nobles; ce sont des altérations parcellaires, sans topographie élective, discrètes. Pas de congestion vasculaire apparente (fig. 1).

Surcharge pigmentaire assez diffuse du parenchyme splénique ; la pulpe blanche est peu visible. Mégacaryocytes peu nombreux.

- Souris morte le 26 novembre (35 jours) = Foie modifié par des images d'hépatite dégénérative; elle affecte une topographie électivement centro-lobulaire ; la périphérie des travées de Remak est relativement conservée. Du point de vue morphologique, on note des images de dégénérescence vacuolaire cytoplasmique, des 
images de lyse totale des éléments, la trame réticulinique des travées persistant seule alors; il y a une réaction interstitielle assez prononcée sous forme d'amas mononucléés, soit au sein des travées,
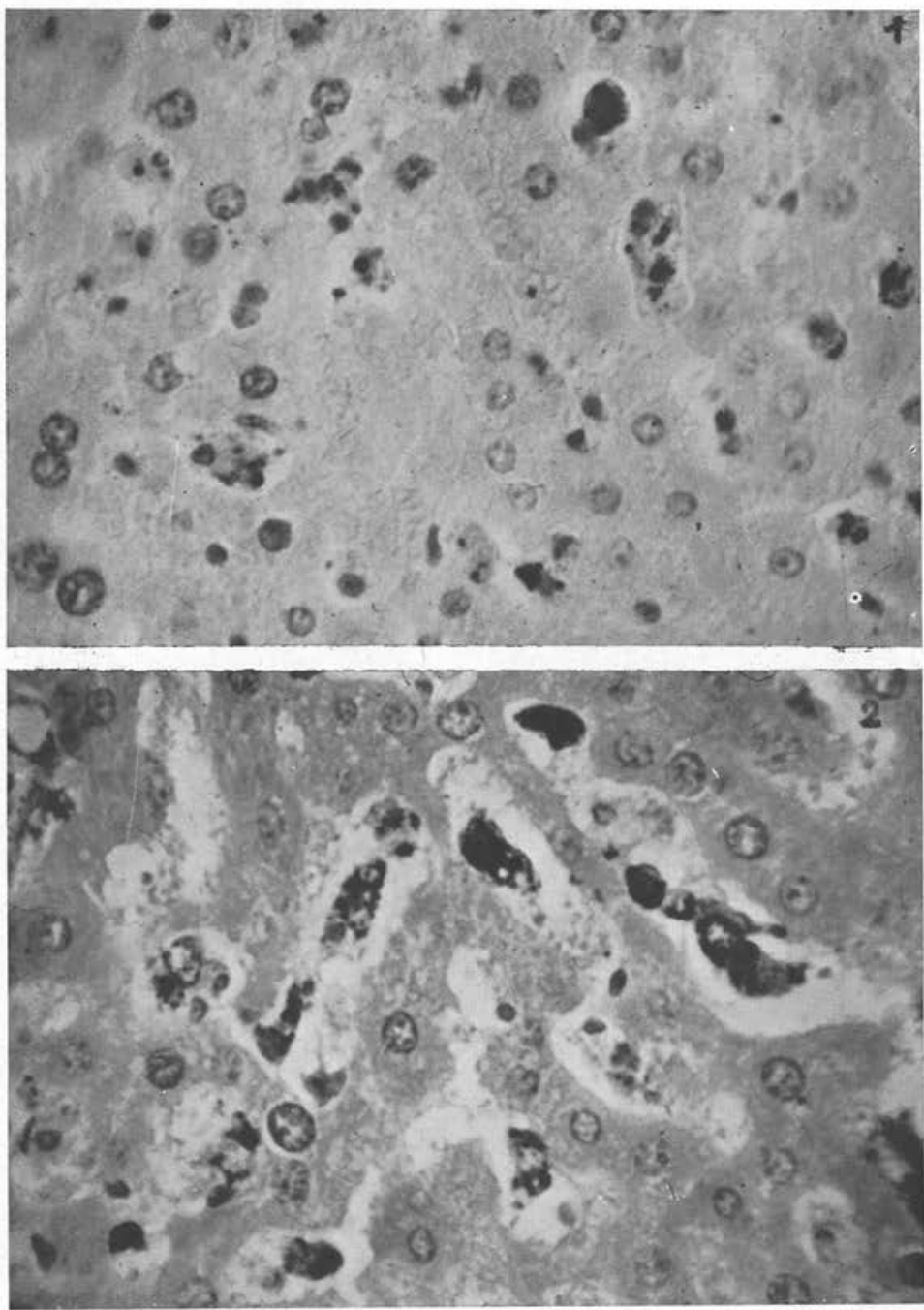

Fig. 2. - (1) 104-6.11 (15 jours). Foie. Surcharge pigmentaire modérée intéressant exclusivement les cellules de Küpfer au sein des capillaires sinusoïdes.

(2) 116-24.11 (33 jours). Foie. Dépôts pigmentaires présentant la même topographie mais beaucoup pius importants que précédemment. 
soit au contact des espaces portes. Il existe enfin une surcharge pigmentaire küppférienne notable intracellulaire.

Rate comportant une réticulose réactionnelle importante au sein
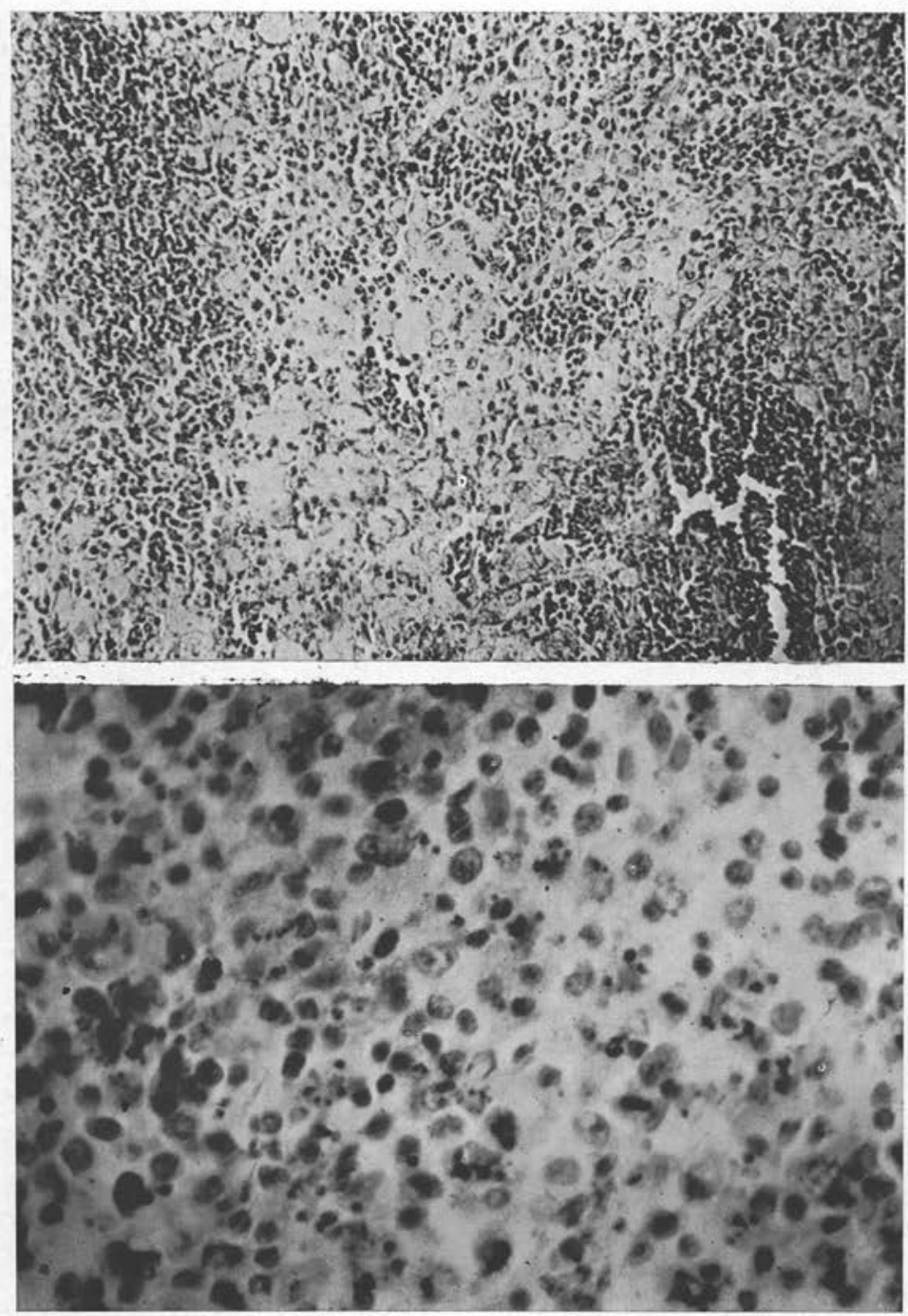

Fig. 3. - (1) 104-30.10 (8 jours). Rate. Congestion importante des sinus de la pulpe rouge.

(2) 104-6.11 (15 jours). Rate. Réaction macrophagique importante au contact du pigment hémomélanique dans la pulpe rouge, sans congestion apparente des sinus. 
de la pulpe rouge; présence de dépôts pigmentaires importants, mais à un degré moindre que dans le foie.

Série 104 : Huile de foie de morue (vitamine A, 600 U.T., et vitamine D, 85 U.T., $0,3 \mathrm{~cm}^{3}$, tous les 2 jours).

— Souris morte le 30 octobre $(8$ jours $)=$ Lésion hépatique moins prononcée que précédemment, se limitant à la surcharge des cellules de Küppfer.

Rate de structure maintenue, mais de caractère congestif important, avec images d'histiocytose et de macrophagie réactionnelle au contact de pigment. Mégacaryocytes peu nombreux (fig. 3).

- Souris morte le 2 novembre (11 jours) = Foie moins altéré que précédemment; on retrouve toutefois des lésions affectant une topographie analogue, à prédominance centro- et médio-lobulaire, la partie périphérique des travées étant indemne. Il s'agit ici essentiellement d'aspects dégénératifs vacuolaires cytoplasmiques; les signes de nécrose cellulaire sont par contre minimes. La réaction interstitielle en amas au sein des travées et au contact des espaces portes est appréciable. Les dépôts pigmentaires sont infiniment moins prononcés, de topographie un peu différente : ils affectent essentiellement des éléments küppfériens et se présentent sous forme de granules isolés, très colorés, brun foncé. Noter la présence de mégacaryocytes dans le parenchyme hépatique.

- Souris morte le 6 novembre (15 jours) = Lésion hépatique se limitant à une surcharge pigmentaire küppférienne discrète et à une réaction mononucléée minime au sein de certains espaces portes (fig. 2).

Réaction macrophagique importante dans le parenchyme splénique; la pulpe blanche est cependant nettement individualisée. Nombreux éléments plasmodiaux du type mégacaryocytaire (fig. 3).

— Souris morte le 11 novembre $(20$ jours $)=$ Foie comportant apparemment peu de lésions parenchymateuses; il existe, par contre, des dépòts pigmentaires importants au sein des cellules de Küppfer, dépôts présentant les mêmes caractères morphologiques que précédemment ; ils se retrouvent dans la rate au niveau de la pulpe rouge.

- Série 116 : Vitamine A, 2.000 U.T., et vitamine D, 500 U.T., $0,3 \mathrm{~cm}^{3}$ tous les 2 jours.

— Souris morte le 3 novembre (14 jours) = Foie : réaction küppférienne importante avec surcharge pigmentaire; les cellules hépatiques sont relativement peu altérées; à retenir cependant 
quelques images de vacuolisation cytoplasmique et un certain degré d'anisonucléose.

Rate présentant des images de réticulose et d'histiocytose importantes au contact de dépôts pigmentaires développés dans l'ensemble de la pulpe rouge.

- Souris morte le 9 novembre (18 jours) = Foie : surcharge pigmentaire prononcée, à prédominance médio-lobulaire; atteinte parenchymateuse représentée par deux foyers nodulaires dégénératifs à cellules lysées, avec nécrose éosinophile du cytoplasme et infiltration à polynucléaires discrète. Réaction mononucléée périvasculaire peu importante ; congestion veineuse assez prononcée.

Rate : réaction macrophagique importante de la pulpe rouge, la pulpe blanche demeurant identifiable. Nombreux éléments plasmodiaux.

- Souris morte le 24 novembre $(3$ jours $)=$ Foie : surcharge pigmentaire importante à prédominance médio-lobulaire ; présence d'une réaction mononucléée au contact des espaces portes ou des veines sus-hépatiques (fig. 4).

Dans la rate, réaction macrophagique diffuse du parenchyme splénique, la pulpe blanche étant relativement peu visible. Mégacaryocytes nombreux.

Série 119: Vitamine A, 2.000 U.T., et vitamine D, 500 U.T., $0,3 \mathrm{~cm}^{3}$ tous les jours.

- Souris morte le 3 novembre (12 jours) = Foie : pas de réaction küppférienne, pas de surcharge pigmentaire; les images de dégénérescence vacuolaire cytoplasmique ne se retrouvent pas.

Rate comportant des signes de réticulose modérée; surcharge pigmentaire minime.

- Souris morte le 7 novembre $(16$ jours $)=$ Foie : surcharge pigmentaire d'intensité moyenne; réaction mononucléée minime au contact de certains vaisseaux.

Rate : réticulose macrophagique diffuse, la pulpe blanche étant peu visible ; mégacaryocytose minime.

- Souris morte le 13 novembre (22 jours) = Foie : surcharge pigmentaire assez marquée, à prédominance médio-lobulaire.

Rate : aspect congestif de la pulpe rouge ; la réaction macrophagique y est développée ; rares mégacaryocytes. Pulpe blanche visible; les corpuscules sont peu volumineux. 


\section{SOURIS AVEC RÉGIMES, IMPALUDÉES, MAIS NON TRAITEES}

Série 105: Huile de foie de morue (vitamine A, 2.000 U.T., et vitamine D, 500 U.T.), $0,3 \mathrm{~cm}^{3}$ tous les jours.

- Souris morte le 30 octobre ( 8 jours $)=$ Foie : dépôts pigmentaires discrets au sein des éléments küppfériens.

Dans la rate, aspect très congestif de la pulpe rouge splénique ; peu de plasmodes ; surcharge pigmentaire et macrophagie.

- Souris morte le 31 octobre ( 9 jours) $=$ Foie comportant peu d'altérations ; absence de dépôts pigmentaires ; absence de réaction interstitielle importante; celle que l'on observe parait constamment retrouvée chez l'animal; pas de signes avérés de souffrance nucléaire ; à noter des images de vacuolisation endo-cytoplasmique des cellules nobles.

- Souris morte le $1^{\text {er }}$ novembre 1956 (10 jours $)=$ Foie relativement moins altéré que dans les séries précédentes ; présence cependant d'une réaction küppférienne au contact de dépôts pigmentaires présents, peu importants; présence de signes de souffrance cellulaire avec images de dégénérescence vacuolaire cytoplasmique.

La rate comporte une congestion prononcée de la pulpe rouge ; on doit retenir d'une part une réticulose réactionnelle assez prononcée, d'autre part la présence d'histiocytes en surcharge pigmentaire.

- Souris morte le 4 novembre (13 jours) = Dans le foie, dépóts pigmentaires assez importants, avec prédominance médio-lobulaire des gros placards; réaction küppférienne associée.

Dans la rate, réticulose réactionnelle et surcharge pigmentaire diffuse de la pulpe rouge; la pulpe blanche comporte des signes d'hyperplasie réticulaire au sein des corpuscules. Mégacaryocytes peu nombreux.

Série 120: Vitamine A, 2.000 U.T., et vitamine D, 500 U.T., $0,3 \mathrm{~cm}^{3}$ tous les jours.

— Souris morte le 30 octobre ( 8 jours) : Foie : surcharge pigmentaire discrète au sein des cellules de Küppfer.

Rate relativement peu congestive ; réaction réticulaire et macrophagique importante. Présence d'éléments mégacaryocytaires. Pulpe blanche individualisée.

- Souris morte le 5 novembre $(14$ jours $)=$ Foie : surcharge pigmentaire d'intensité moyenne avec réaction küppférienne et à prédominance médio-lobulaire discrète. Réaction mononucléécs périvasculaire minime. 
Rate : réticulose réactionnelle de la pulpe rouge, mais avec surcharge pigmentaire discrète; participation de la pulpe blanche à la réticulose.

- Souris morte le 8 novembre (17 jours) = Dans le foie : lésions importantes intéressant essentiellement les éléments küppfériens ; la surcharge pigmentaire est massive; infiltrats inflammatoires interstitiels péri-portaux ; vaisseaux congestifs. Par contre, peu de modifications des cellules nobles, en dehors d'une anisocaryose appréciable.

Rate : surcharge pigmentaire importante et diffuse de la pulpe rouge ; la réaction réticulaire développée à son niveau est relativement peu importante.

- Souris morte le 9 novembre $(18$ jours $)=$ Foie : surcharge pigmentaire prononcée; les placards les plus importants se situent dans la zone médio-lobulaire; présence de deux nodules d'aspect scléro-inflammatoire, riches en fibroblastes, apparemment sans relation avec le paludisme ; leur origine ne peut être précisée. Amas mononucléés péri-vasculaires.

Rate comportant une réaction macrophagique importante avec participation de la pulpe blanche. Réaction mégacaryocytaire minime.

\section{SOURIS SANS REGIME, IMPALUDEES ET TRAITEES}

Série 109 : Pain et eau.

- Souris morte le $1^{\text {er }}$ novembre $(10$ jours $)=$ Dans le foie, élément congestif et petits foyers inflammatoires dans le parenchyme sous forme d'îlots à cellules rondes péri-portaux; il faut tenir compte de l'existence d'une parasitose intra-hépatique. Les dépôts pigmentaires dans les cellules de Küppfer sont minimes.

Dans la rate, dépòt pigmentaire parenchymateux, minime ; congestion nette des sinus veineux. La pulpe blanche est nettement individualisée.

- Souris morte le 7 novembre (16 jours) = Dépôts hémomélaniques d'intensité modérée au sein des capillaires sinusoïdes et dans le parenchyme splénique.

Série 100: Pain et eau.

- Souris morte le 12 novembre $(21$ jours $)=$ Dans le foie, congestion veineuse dans le parenchyme hépatique (branches de la veine sus-hépatique et de la veine porte) ; congestion discrète des capillaires sinusoïdes; dépôts pigmentaires importants dans les cellules de Küppfer, sans répartition élective. 
Dans la rate, surcharge pigmentaire assez diffuse dans la pulpe rouge, avec histiocytose réactionnelle à son contact.

- Souris morte le 22 novembre (31 jours) = Dans le foie, surcharge pigmentaire très prononcée dans les cellules de Küppfer, sans répartition élective au sein des travées de Remak; la surcharge pigmentaire est diffuse ; on note de petits îlots lymphocytaires péri-portaux.

Dans la rate, surcharge pigmentaire prononcée de la pulpe rouge ; pulpe blanche mal individualisée.

$$
* *
$$

Tels sont les résultats histologiques obtenus.

$\mathrm{Si}$ nous les comparons selon les deux tableaux joints: d'une part, le tableau I (lésions comparées selon le régime des souris et le traitement; poids, parasitémie et mortalité comparés des divers lots de souris au $22^{\circ}$ jour après l'inoculation), et, d'autre part, le tableau II (lésions comparées en fonction du temps de vie de la souris, donc de la durée de l'infection), il nous semble qu'on puisse faire les remarques suivantes :

$1^{\circ}$ Toutes les souris examinées étant mortes à partir du $8^{\circ}$ jour, nous nous trouvons en présence du $2^{\circ}$ stade de la maladie.

$2^{\circ}$ Comparativement avec les observations anatomo-pathologiques faites par divers auteurs, et en particulier S. Hadjères et J. Orfila (1955), nous avons retrouvé les éléments d'une surcharge küppférienne en hémomélanine, surcharge intéressant électivement le S.R.H. et à laquelle participe la rate.

Les autres altérations nous ont paru (contrairement aux constatations antérieurement citées) inconstantes et de caractère contingent.

Ainsi en est-il de la congestion vasculaire, qui n'est pas évidente au niveau des capillaires sinusoïdes, et des atteintes "parenchymateuses », les lésions de la cellule hépatique n'étant retrouvées que rarement ; elles ont un caractère parcellaire : sont-elles en relation directe avec l'infection paludéenne ? nous n'oserions l'affirmer: celle-ci, à notre sens, est représentée essentiellement par la réaction réticulaire hépato-splénique.

$3^{\circ}$ L'apport d'huile de foie de morue contribue-t-il à provoquer des altérations dégénératives des cellules nobles? Nous avons observé sur onze animaux : deux cas d'altérations appréciables et trois à caractère parcellaire et discret $(5 / 11)$. Il faut remarquer, du reste, qu'en l'absence de tout véhicule huileux, des altérations ont 


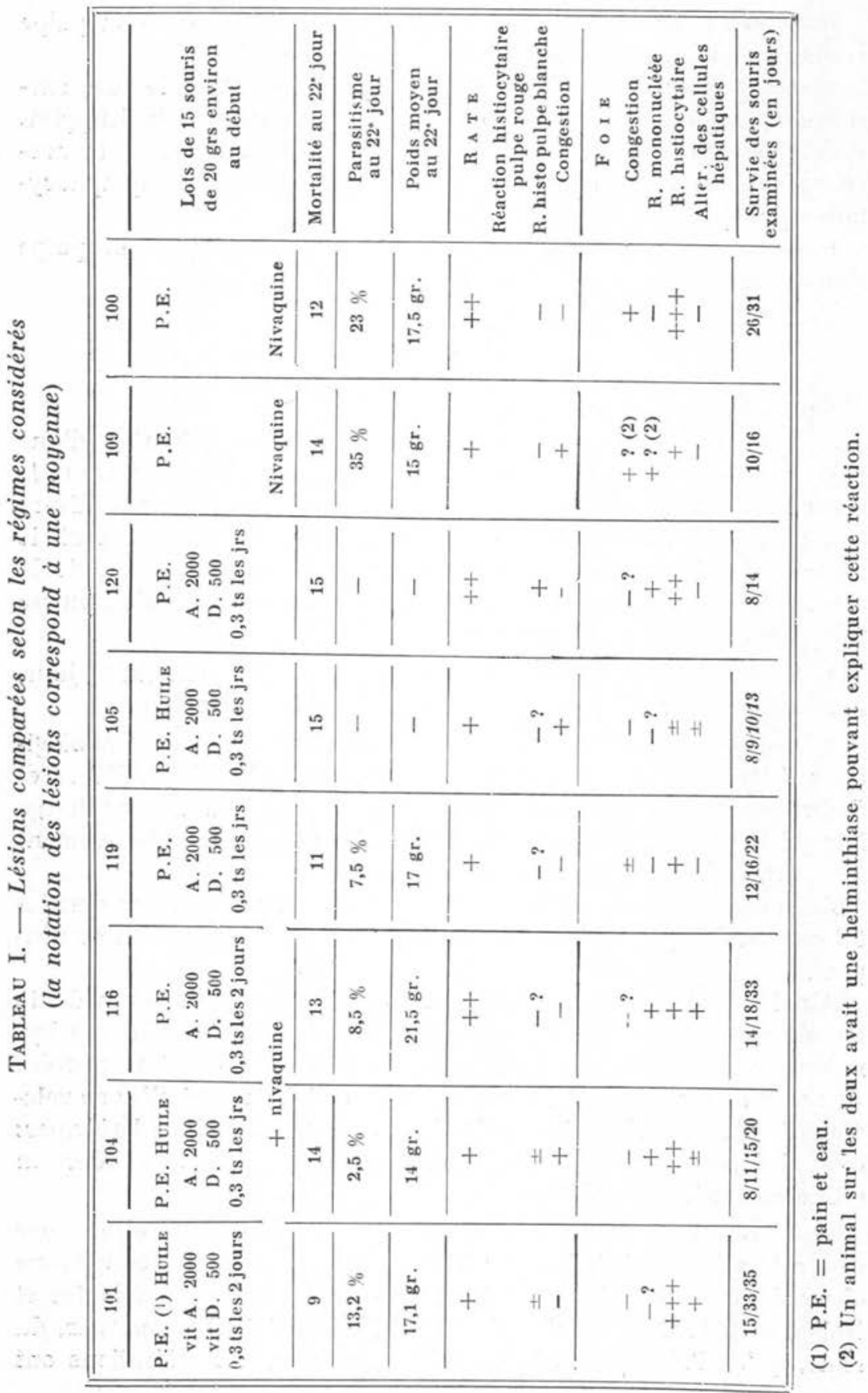


TABleau II

Lésions comparées en fonction de la durée de l'infection

\begin{tabular}{|c|c|c|c|c|c|c|c|c|}
\hline \multirow{3}{*}{$\begin{array}{c}\mathrm{N}^{\cdot} \\
\text { SÉRIE } \\
\text { DES } \\
\text { SOURIS }\end{array}$} & \multirow{3}{*}{$\begin{array}{l}\text { SURVIE } \\
\text { ( } \mathrm{K} \text {. } \\
\text { JOURS) } \\
\text { APRÈS } \\
\text { L'INOCU- } \\
\text { LATION }\end{array}$} & \multirow{2}{*}{ 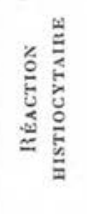 } & \multirow{2}{*}{ 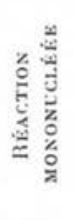 } & \multirow{2}{*}{ 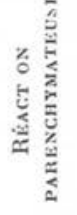 } & \multirow{2}{*}{ 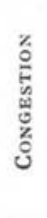 } & \multicolumn{3}{|c|}{ RÉaction histiocytaire } \\
\hline & & & & & & $\begin{array}{l}\text { Pulpe } \\
\text { rouge }\end{array}$ & $\begin{array}{c}\text { Pul, e } \\
\text { blanche }\end{array}$ & 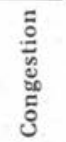 \\
\hline & & \multicolumn{4}{|c|}{ FO I E } & \multicolumn{3}{|c|}{ R A T E } \\
\hline 104 & 8 & + & \pm & - & - & + & - & ++ \\
\hline 105 & 8 & + & - & - & - & + & - & ++ \\
\hline 120 & 8 & + & - & - & - & + & - & \pm \\
\hline 105 & 9 & - & - & \pm & - & - & - & - \\
\hline 105 & 10 & + & + & + & - & + & - & ++ \\
\hline 104 & 10 & + & - & + & - & - & - & - \\
\hline 109 & 10 (Hel) & - & + & - & + & - & - & + \\
\hline 119 & 12 & - & - & - & - & \pm & - & - \\
\hline 105 & 13 & ++ & - & - & - & + & + & - \\
\hline 120 & 14 & ++ & \pm & - & - & + & + & - \\
\hline 116 & 14 & ++ & - & \pm & - & + & - & - \\
\hline 101 & 15 & ++ & - & - & - & + & - & - \\
\hline 104 & 15 & ++ & + & - & - & + & + & - \\
\hline 119 & 16 & ++ & \pm & - & - & + & + & - \\
\hline 109 & 16 & + & - & - & - & + & - & - \\
\hline 120 & 17 & ++ & + & - & + & + & - & - \\
\hline 116 & 18 & ++ & + & + & - & + & \pm & - \\
\hline 120 & 18 & ++ & + & $+?$ & - & + & + & - \\
\hline 104 & 20 & + & - & - & - & + & - & - \\
\hline 100 & 21 & ++ & - & - & + & + & - & - \\
\hline 119 & 22 & ++ & - & - & - & + & - & - \\
\hline 100 & 31 & +++ & - & - & - & + & - & - \\
\hline 101 & 33 & +++ & - & \pm & - & + & \pm & - \\
\hline 116 & 33 & +++ & + & - & - & + & - & - \\
\hline 101 & 35 & +++ & + & ++ & - & + & - & - \\
\hline
\end{tabular}


été également observées : une réaction appréciable dans un cas et deux minimes sur 10 animaux $(3 / 10)$.

Ces manifestations sont inconstantes, la différence entre les deux groupes n'est pas significative, et nous ne pouvons ni affirmer, ni infirmer notre hypothèse.

$4^{\circ}$ Il nous paraît intéressant de signaler que les modifications des lésions en fonction du facteur temps sont, par contre, beaucoup plus nettes, abstraction faite des conditions expérimentales. Ces modifications moins apparentes, dans l'ensemble, au niveau du parenchyme splénique, sont plus caractéristiques au niveau du foie. Notons cependant que c'est dans la rate, à des délais relativement précoces (8-10 jours), que l'on observe un élément congestif important de la pulpe rouge, alors qu'il est peu ou pas évident dans le foie.

Par contre, l'intensité des réactions histiocytaires liées à la surcharge pigmentaire hémomélanique, est nettement proportionnelle, dans le parenchyme hépatique, à la durée de survie des animaux.

\section{RÉsu mÉ}

L'examen anatomo-pathologique des foies et des rates de souris mortes au stade II d'infection à $P l$. berghei n'a pas permis de constater de différences appréciables, qu'il y ait l'adjonction ou non d'un régime vitaminé (A-D) en suspension aqueuse ou huileuse.

Les seules modifications observées portent sur l'intensité de la réaction histiocytaire en présence du parasite, directement proportionnelle à la durée de la survie des animaux.

Il ne nous semble pas, au stade II, que la congestion vasculaire constitue un élément marqué du tableau pathologique.

(Travail du Laboratoire d'Anatomie pathologique et de l'Institut d'Hygiène tropicale et de Médecine sociale Faculté de Médecine, Marseille)

\section{BiBLIOGRAPHIE}

Orfila (J.), 1955. - Le Paludisme expérimental de la souris blanche. Thèse, Faculté de Médecine d'Alger.

Sautet (J.), Desanti (J.), Vuillet (J.), Arnaud (G.) et Ausseil (M.), 1957. - Précisions sur l'adjuvant renforçateur du terrain le plus favorable dans les traitements courts et à faibles doses par un amino-4-quinoléine, dans les affections à Pl. Berghei chez la souris blanche. Bull. Soc. Path. Exot., 1, p. 49.

Van Brand (T.) et Mercado (T. L.), 1958. - Quantitative and histochemical studies on lever lipides of rats infected with Pl. Berhei. Ann. J. Hyg., 67 , p. 31 . 